\title{
Clinicoradiological Approach to Sonomammographic Spectrum of Breast Disorders
}

\author{
${ }^{1}$ Vandana V Ahluwalia, ${ }^{2}$ Prerna S Saharan, ${ }^{3}$ Ankita Chauhan
}

\begin{abstract}
The current cancerophobic climates surrounding breast disease affect the sequence of imaging modalities and follow-up. Neither the physician nor the patient can be sure that the palpable lump is an actual mass or an unexceptional variant and, here is the active/decisive role of a radiologist to evaluate sonomammographic appearances as per Breast Imaging Reporting and Data System Lexicon, and then assuring the patient for its benignity or asserting the necessity of further intervention. This study reviews key sonomammographic features of common and unusual benign breast disease, infections, and systemic disorders affecting breast in the female patient.
\end{abstract}

Keywords: Apocrine metaplasia, Asymmetry, Breast imaging reporting and data system, Infection, Sonomammography.

How to cite this article: Ahluwalia VV, Saharan PS, Chauhan A. Clinicoradiological Approach to Sonomammographic Spectrum of Breast Disorders. Donald School J Ultrasound Obstet Gynecol 2017;11(2):126-134.

Source of support: Nil

Conflict of interest: None

\section{INTRODUCTION}

Breast-related symptoms are one of the most common reasons for visiting a physician by women of all age groups. However, a majority of breast symptoms or lesions will prove to be of benign etiology. Physical, psychological, and financial costs of investigating benign breast disease, primarily to exclude malignancy, are substantial, but patient satisfaction is always a priority.

Evaluating a breast mass is difficult for the patient because of anxiety associated with fear of breast malignancy and challenging for the physician to be confident of the fact that he/she is palpating an actual mass or a variant of normal breast parenchyma and here is the active/decisive role of a radiologist. It is the radiologist who evaluates the content and significance of nodular density, the degree of suspicion for it to be malignant lesion and assuring the patient for its benignity or

${ }^{1}$ Professor, ${ }^{2}$ Resident, ${ }^{3}$ Assistant Professor

${ }^{1-3}$ Department of Radiodiagnosis, Sarojini Naidu Medical College, Agra, Uttar Pradesh, India

Corresponding Author: Prerna S Saharan, Resident, Department of Radiodiagnosis, Sarojini Naidu Medical College, Agra, Uttar Pradesh, India, e-mail: prernaspmc@gmail.com asserting the clinician/patient necessity of undergoing fine needle aspiration cytology. Beyond the role of imaging, the radiologist also plays a role of the reassuring physician to allay fear and can advise the further management.

The study presents our clinical experience of sonomammographic findings over a period of more than 15 years based on lesion morphology, Breast Imaging Reporting and Data System (BI-RADS) criteria, cytological, operative, and histopathological correlation of the common and few rare benign breast lesion and infections.

\section{SONOMAMMOGRAPHIC CORRELATION}

Mammography has been a well known and many times the first imaging modality to evaluate a breast pathology. The standard views are craniocaudal (CC) and mediolateral view (MLO); however, more projections can be added for further characterization of a mass lesion. Ultrasonography (USG) depicts relative difference in the density and acoustic impedance of the lesion respectively, as compared with the surrounding breast tissue and remains the first modality of choice in young patients with palpable lesions.

Sonography and mammography are now widely used concurrently in evaluating breast pathology for reporting a final BI-RADS category. Sonography has an additional advantage in assessing a mammographic abnormality ranging from discrete mass to focal or global asymmetric densities especially when not associated with suspicious calcification. With the increase in mammographic detection of smaller nonpalpable lesions, it is sometimes challenging to confirm the subsequent localization of the lesion on sonography, but by using a retractable localization wire or a biopsy marker clip we can demarcate the lesion for better sonomammographic correlation. ${ }^{1,2}$

The mammographic-sonographic correlation in size, shape, location, and surrounding tissue is to be made using standard Lexicon guidelines carefully taking into account the differences encountered while using these modalities concurrently.

On mammography, the dilated duct and intraductal lesions can appear as subareolar tortuosities; however, the high-resolution ultrasound (US) more reliably defines the extent of retroareolar ducts, intraductal soft tissue, and vascularity. The USG should be the modality in 
patients with nipple discharge with or without mammographic abnormality. Breast density is an increasingly opportune issue in breast cancer diagnosis. Breast density causes a decrease in the sensitivity of mammography for cancer detection, with a significant increase in the breast cancer risk. ${ }^{3}$

\section{BENIGN LESIONS}

Cysts (Fig. 1) are the commonest cause of breast lumps in women between 35 and 50 years of age. ${ }^{4}$ Mammography shows a well-defined hypo- to isodensity lesion; however, the cystic nature of the lesion is only seen in USG. There are two types of cysts: Secretory (apocrine) and transudative (retention) cyst. ${ }^{4}$ A secretory cyst results from active apocrine cells which can undergo metaplasia and is premalignant. Transudative cyst is a retention cyst which response well to aspiration. A single cyst is more likely to be retention cyst, whereas an apocrine cyst is known to be multiple. To be called a simple cyst, the cyst must satisfy all Stavros's sonographic criteria $^{5,6}$ : Anechoic, well-circumscribed with a thin echogenic capsule, increased ultrasonic transmission, and thin-edge shado A complex cyst is a mixed echogenic or heterogeneous hypoechoic lesion with a thick wall and thick septa (>5 mm), and also shows a good response to aspiration. Cysts can be followed up on USG with patient counseling.

Fibroadenoma is the most common lesions encountered in day-to-day practice, $91 \%$ of all solid breast masses being in girls ( $<19$ years). ${ }^{7}$ They can be single or multiple and of variable size (Fig. 2). On mammogram, oval or lobulated circumscribed mass with dense, coarse calcifications $(\mathrm{Ca}++)$ was present. Ultrasound features are hypoechoic circumscribed oval mass with homogeneous internal echoes. A giant fibroadenoma is a fibroadenoma over 5 to $10 \mathrm{~cm}$ diameter (Fig. 3). It shows features of benign nature; however, indeterminate lesions can require pathological evaluation. Growth $>20 \%$ in diameter in 6 months suggests possible phyllodes and needs excision.

Phyllodes tumor or cystosarcoma phyllodes (Fig. 4) is a rare fibroepithelial neoplasm that share many clinical, pathologic, and imaging features with giant fibroadenoma. There is an increased incidence of phyllodes tumors in people of Asian heritage. ${ }^{8}$ It usually reveals clear margins
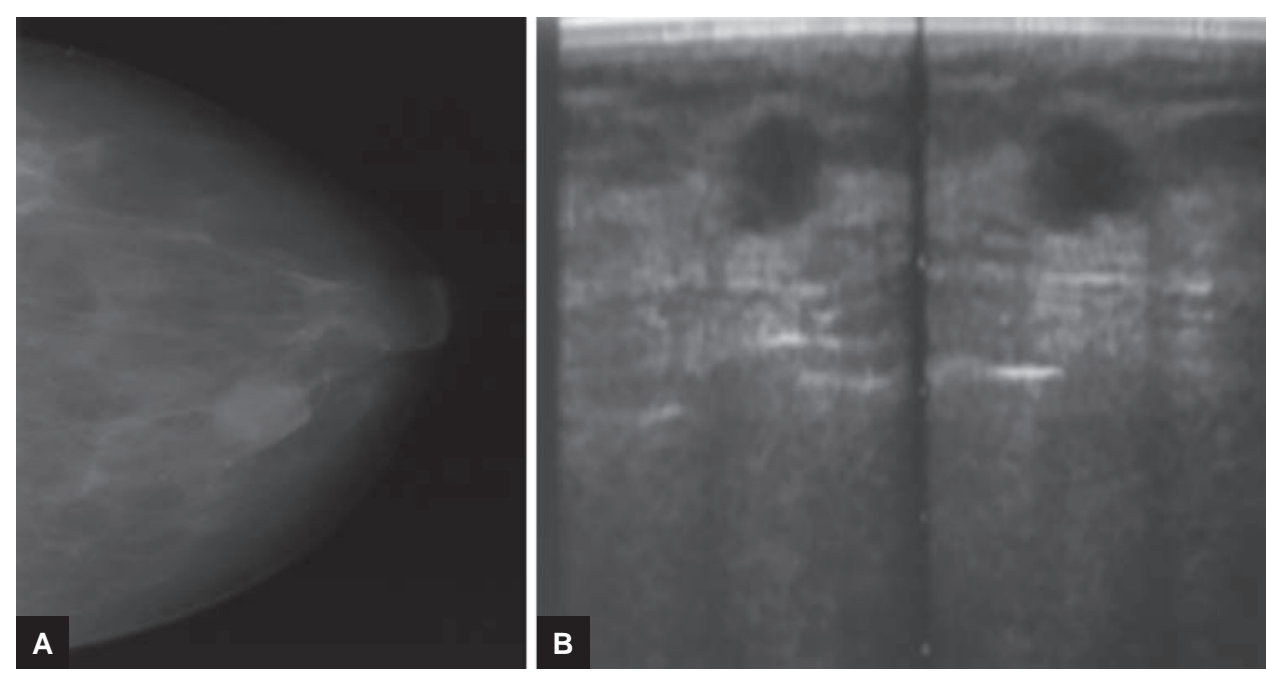

Figs $1 \mathrm{~A}$ and B: Simple cyst - CC mammogram: (A) Shows iso- to hyperdense defined lesion with corresponding ultrasonography; and (B) showing well-defined oval cystic lesion with posterior acoustic enhancement
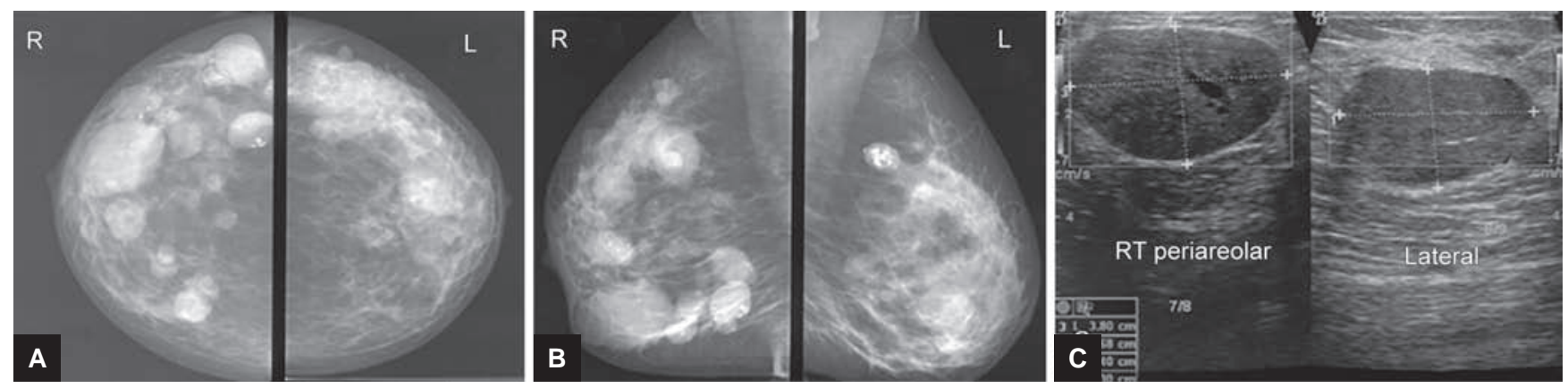

Figs 2A to C: (A) Multiple fibroadenomas - MLO, (B) CC view mammogram showing multiple defined homogenous hyperdense lesions (thin arrows) with few showing coarse dystrophic calcification (thick arrow), (C) Sonogram with well-defined hypoechoic lesion 

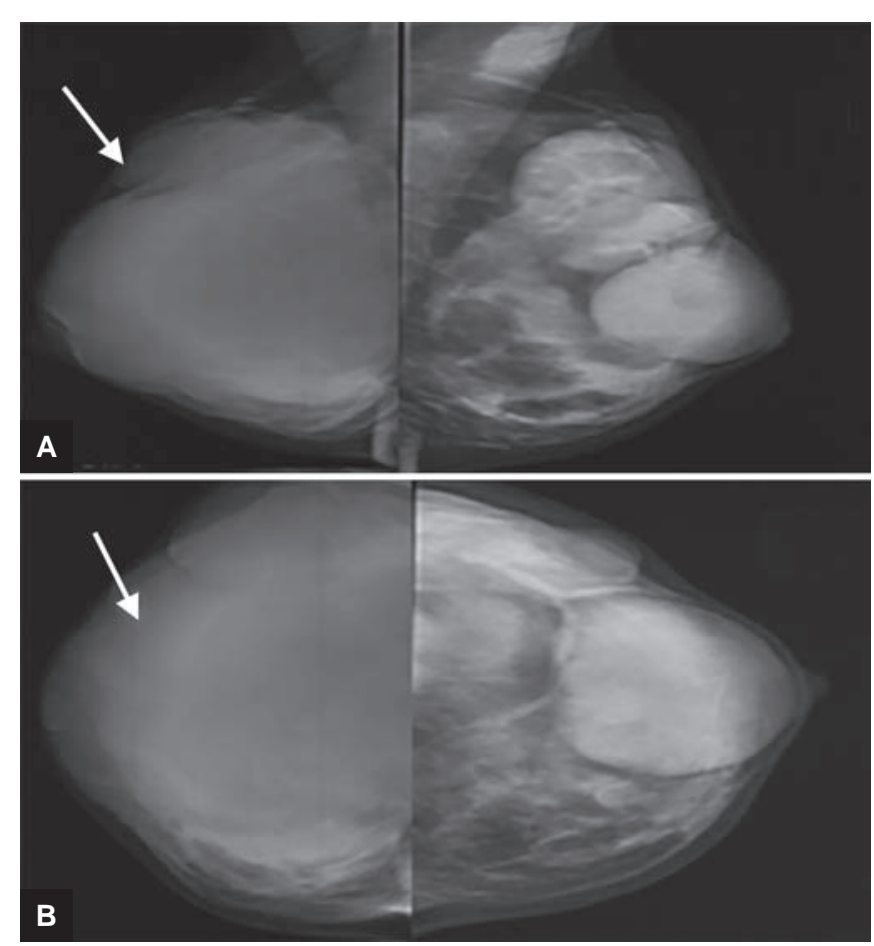

Figs $3 \mathrm{~A}$ and $\mathrm{B}$ : Mammogram shows giant fibroadenoma (arrow) on right displacing parenchymal rim and enlarging the breast contour and multiple fibroadenoma on left side: (A) MLO; (B) CC

and an inhomogeneous echostructure, sometimes with variable cystic areas. Malignant potential is low. Recurrence rates correlates with tumor biology, with benign phyllodes showing a lower recurrence rate (10-25\%), and malignant ones having up to $40 \%$ recurrence rate. ${ }^{8}$

Fibroadenomatoid change (Fig. 5) is a benign proliferative lesion differing from fibroadenoma in involving several lobules, and the stromal hyperplasia may not have well-defined borders. Ultrasonographic findings are more accurate with circumscribed lobulated mixed hypo- and hyperechoic mass with internal echogenic septations. It represents an intermediate step in the histogenesis of fibroadenoma. The fibroadenomatoid nodular lesion thus formed later coalesces to form a fully developed fibroadenoma.

Apocrine metaplasia has acinar dilatation, lined by columnar type secretory epithelium with granular, eosinophilic cytoplasm. Benign conditions like fibrocystic changes, sclerosing adenosis, fibroadenoma, as well as malignancy can show apocrine metaplasia. Apocrine metaplasia in fibroadenoma (Fig. 6) presents as cystic foci within fibroadenoma which on cytology show apocrine metaplasia in ductal epithelium. On USG, these cysts may be small and beyond resolution appearing as small echogenic foci with comet tail artifact. The presence of apocrine metaplasia is not believed to elevate the risk for breast cancer. ${ }^{9,10}$ It is not a premalignant condition but microcalcification on mammography and its association with malignancy warrants further pathological evaluation.

Lipoma is a common benign tumor, i.e., composed of mature adipocytes and is most often unilateral and solitary. ${ }^{11}$ Typically manifesting as a palpable, compressible soft mass on palpation, and on USG, it can be homogeneously hypoechoic, isoechoic, or hyperechoic and has no associated internal or peripheral vascularity. Mammography shows a uniformly radiolucent and smoothly marginated mass without intrinsic density (Fig. 7). If a well-delineated radiolucent mass is echogenic on USG, it is benign, and biopsy is not necessary. ${ }^{12}$ Lipomas
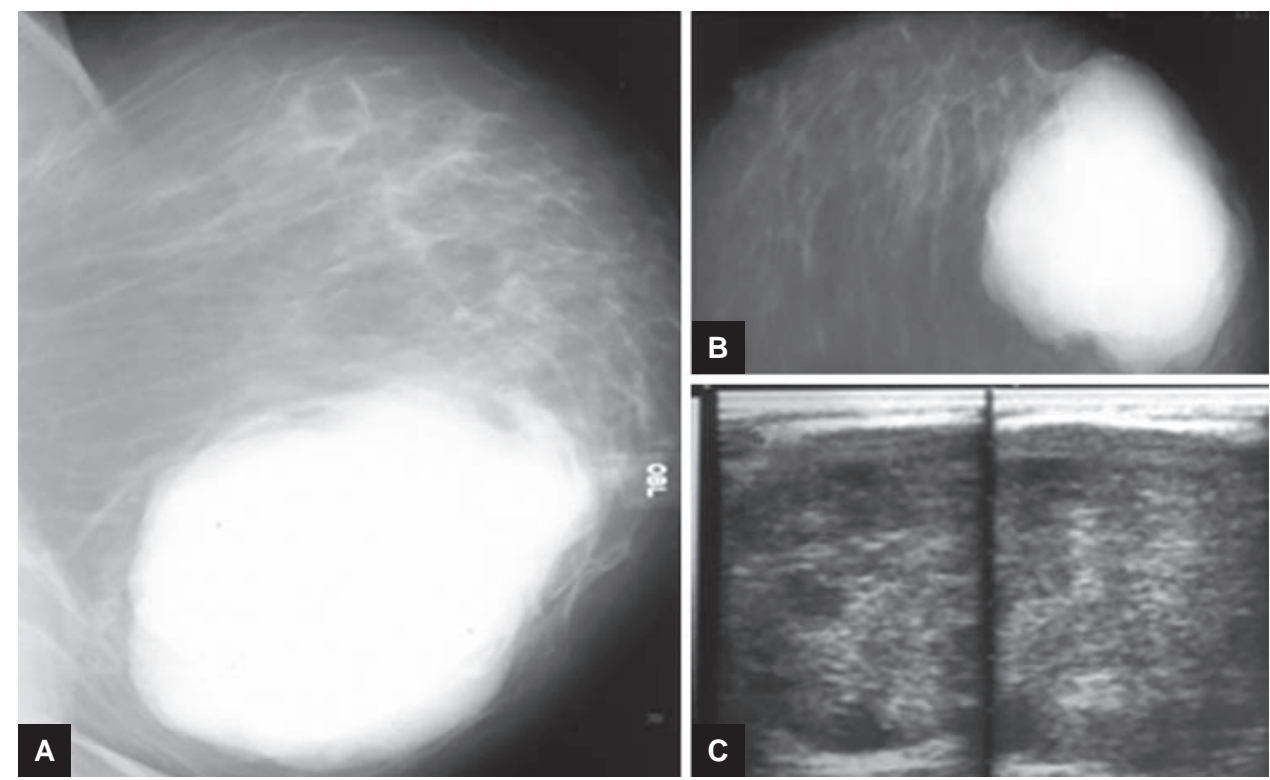

Figs 4A to C: Phyllodes tumor: ( $A$ and $B$ ) Mammogram showing hyperdense lobulated defined mass in the inferior lateral quadrant with a corroborative ultrasound; and (C) showing well-encapsulated lesion with internal heterogeneity and cystic changes 

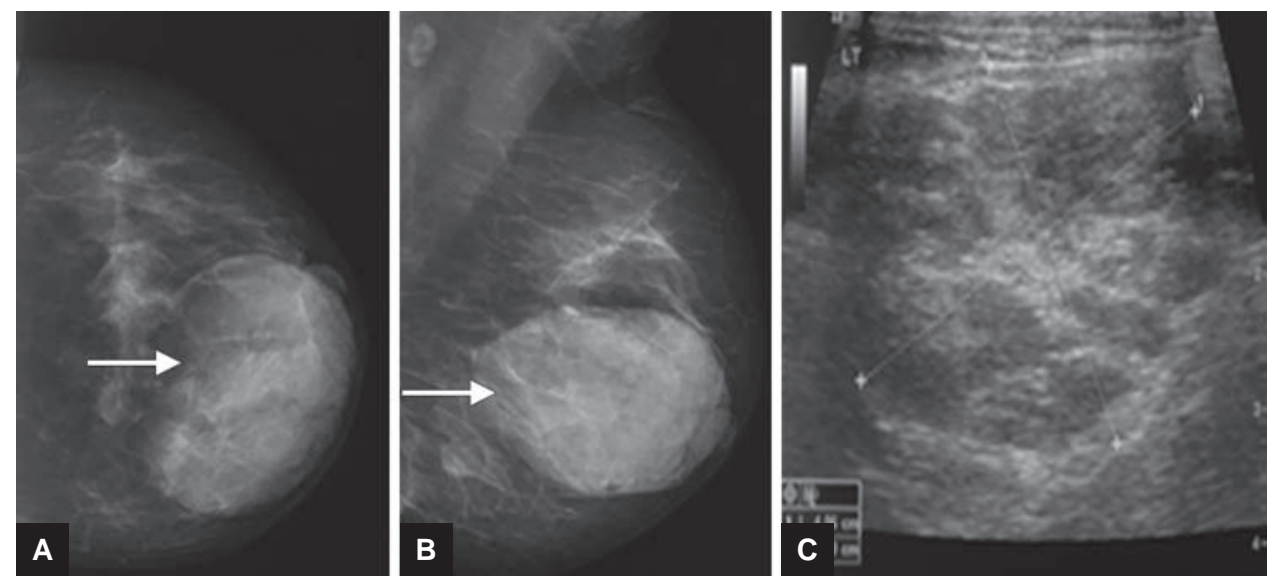

Figs 5A to C: Fibroadenomatoid changes in adenoma-CC: (A) and MLO; (B) view of the mammography showing linear hypodensities in otherwise well-defined hyperdense lesion with corresponding ultrasonography; and (C) showing heterogeneous capsulated lesion with echogenic septation
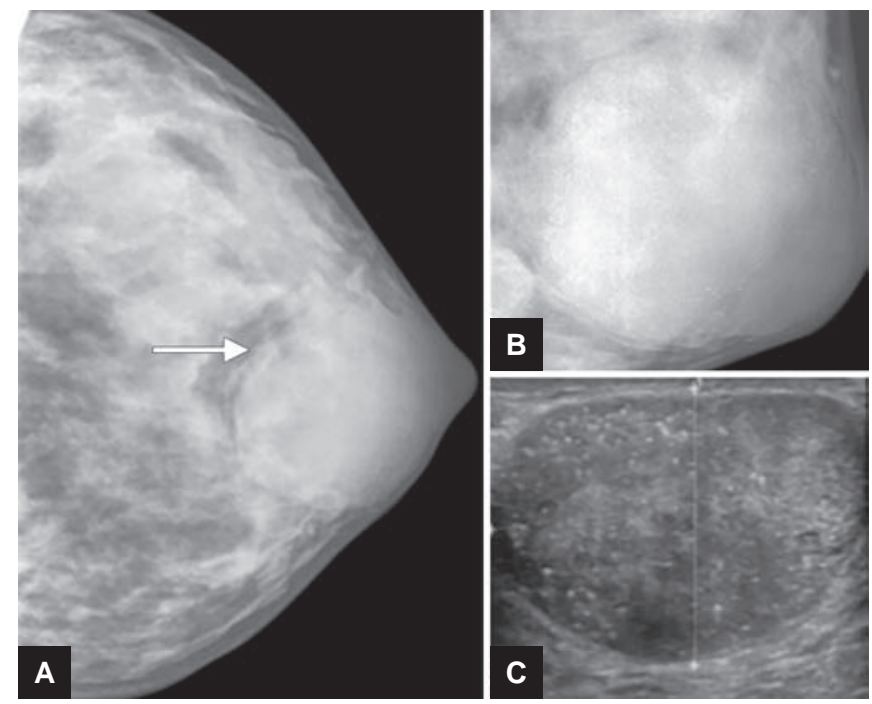

Figs 6 A to $C$ : Apocrine metaplasia in fibroadenoma - CC: $(A)$ and magnified view; (B) of mammogram showing speckled and scattered fine calcific densities in otherwise homogeneously hyperdense retroareolar fibroadenoma and ultrasound; and $(\mathrm{C})$ confirming multiple anechoic cysts showing bright echogenic foci with comet tail artifact giving heterogeneity to the adenomatous lesion can increase in size in response to hormonal stimulation. ${ }^{12}$ Enlarging or symptomatic lipomas should undergo surgical excision. Other fat-containing lesions like galactoceles (Figs 8 and 9) in different stages of evolution shown in the figure and can validate with the clinical history of lactational physiological derangements.

Fibroadenolipoma or hamartoma (Fig. 10) has a capsule with fat and glandular element proliferation and best defined as "Breast-within-a-breast" appearance on a mammogram. On mammography, round or oval circumscribed lesion with varying mixtures of prominent fat and fibroglandular tissue elements was present. It may be uniformly dense if minimal fat present. Pseudocapsule, a rim of compressed parenchyma is often visible. Benign appearing calcifications may be present. On USG mixture of sonolucent fat and echogenic glandular elements gives heterogeneous internal echogenicity. It is circumscribed oval shape with pseudocapsule having variable echogenicity. It is compressible, deformable with pressure, and nontender on USG examination.
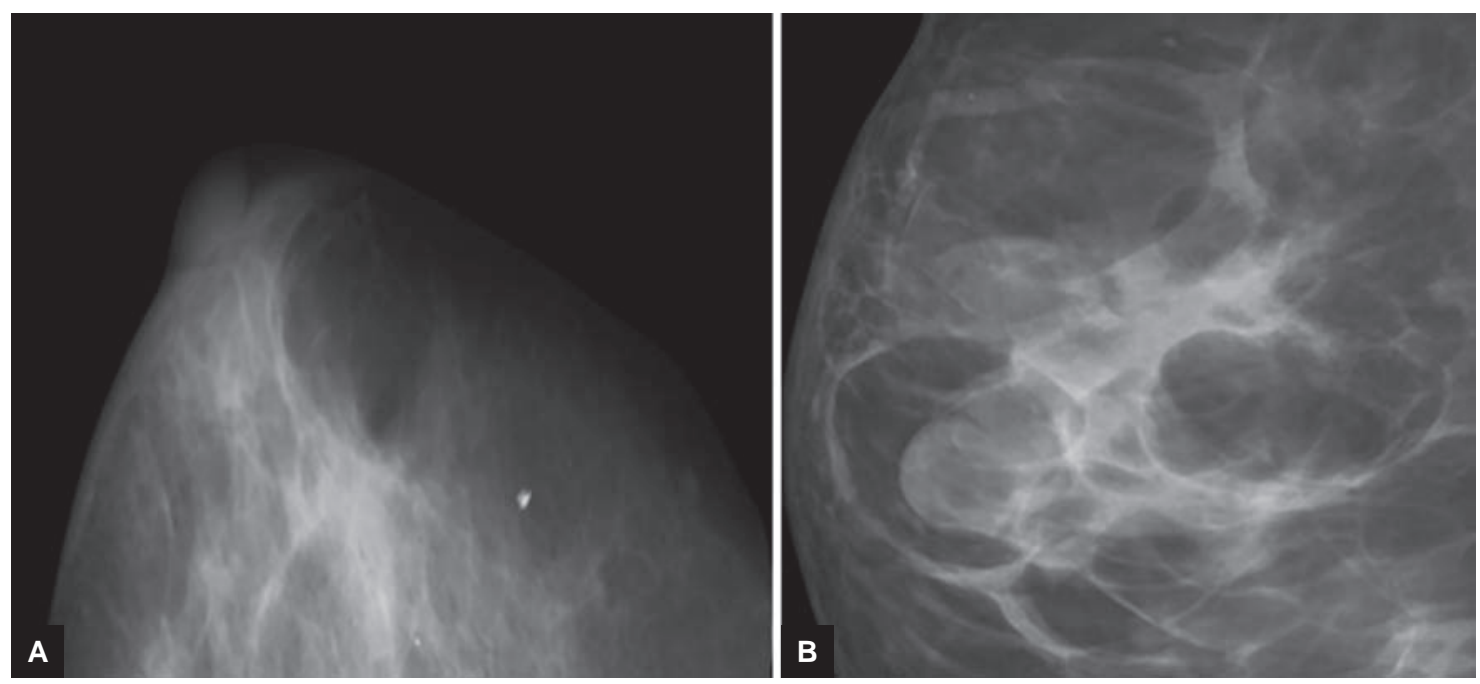

Figs 7A and B: Lipoma - CC mammogram in two different patients presenting as classical lobulated compressible lucency with defined margins seen as single (image 1) and multiple lesions in another patient 

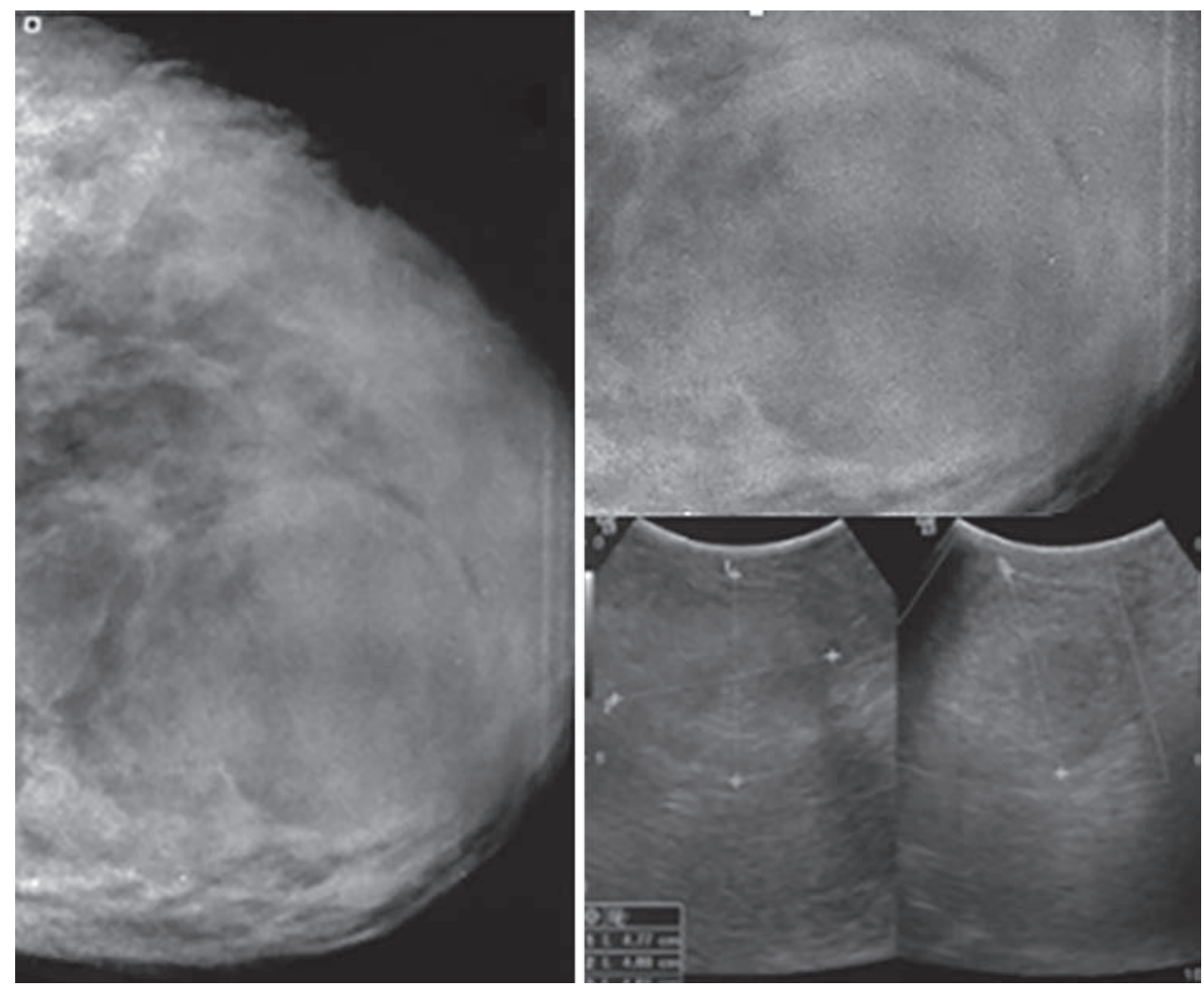

Fig. 8: Early galactocele - MLO view of mammogram showing iso-hypodense lobulated soft tissue marginated by fatty lucency from adjacent glandular lactating breast with corresponding ultrasonography showing nontender echogenic fat density lesion with mobile coarse echoes within without any significant intra or periregional vascularity thus differentiating from inflammatory/infective changes
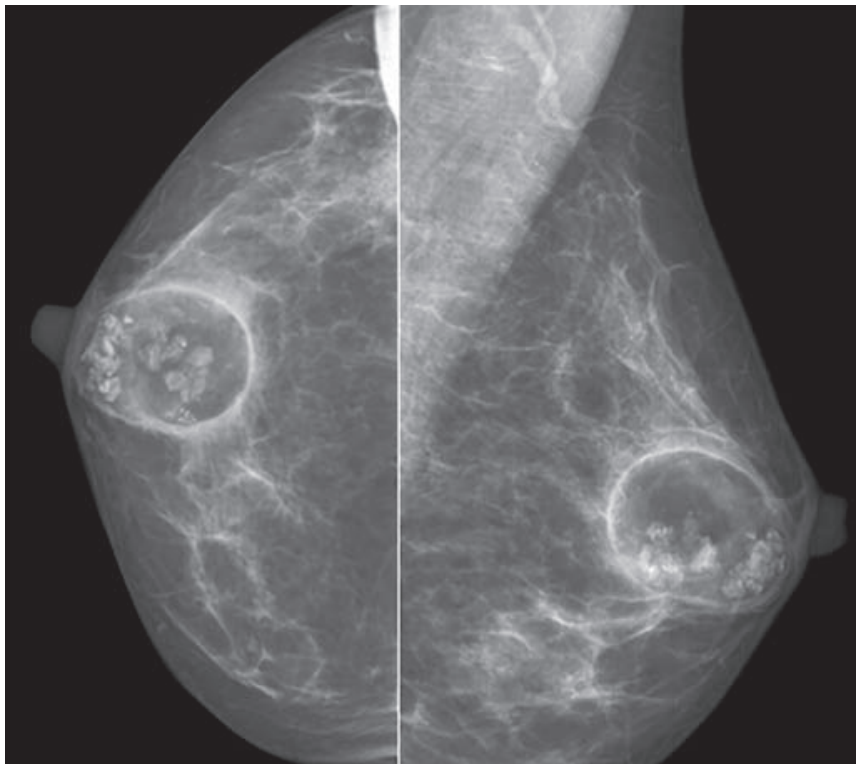

Fig. 9: Chronic galactocele - CC and MLO mammogram view showing retroareolar lucent/fatty lesion with echogenic calcific rim and intraregional hyperdensities with variable calcific foci related to inspissated milk secretions. It was persistent on follow-up

Fat necrosis represents nonsuppurative fatty inflammation related to an injury. Correlation with the clinical history is the key to correct diagnosis. The sonographic appearance of fat necrosis is variable; at times, it can appear echogenic. The mammographic appearance ranges from radiolucent

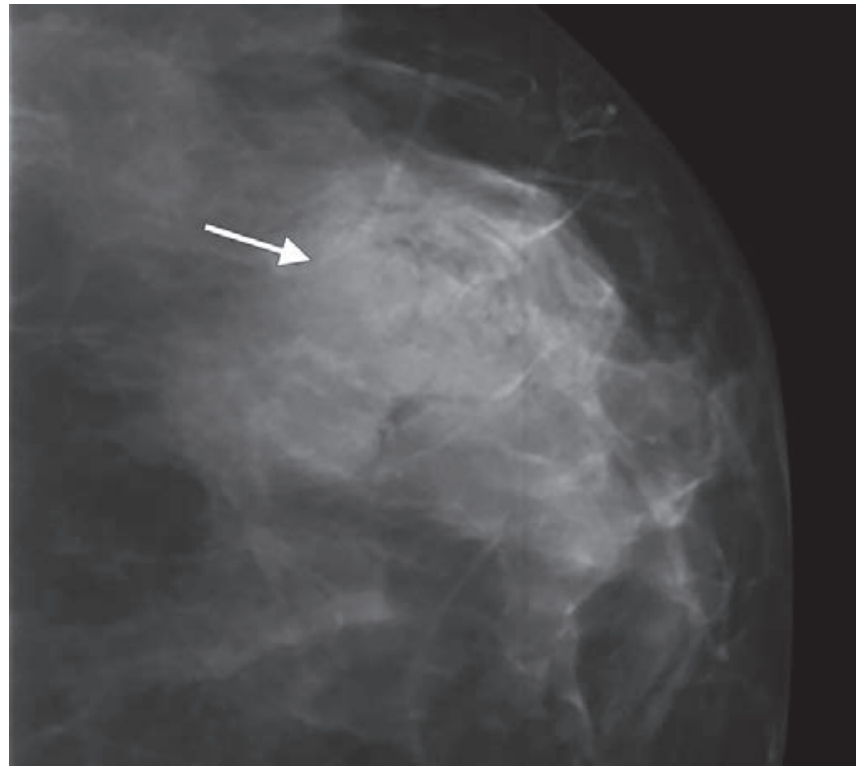

Fig. 10: Fibroadenolipoma - magnified view of mammogram showing lobulated dense lesion (arrow) and scattered fatty lucencies within

areas that may have associated coarse dystrophic calcifications to an irregular, distorted, or a spiculated mass. The abnormal area may become cystic, resolve or decrease in size, or remain stable over time. Fat necrosis $\mathrm{Ca}++$ coarsen over time suggestive of its dystrophic in nature. 

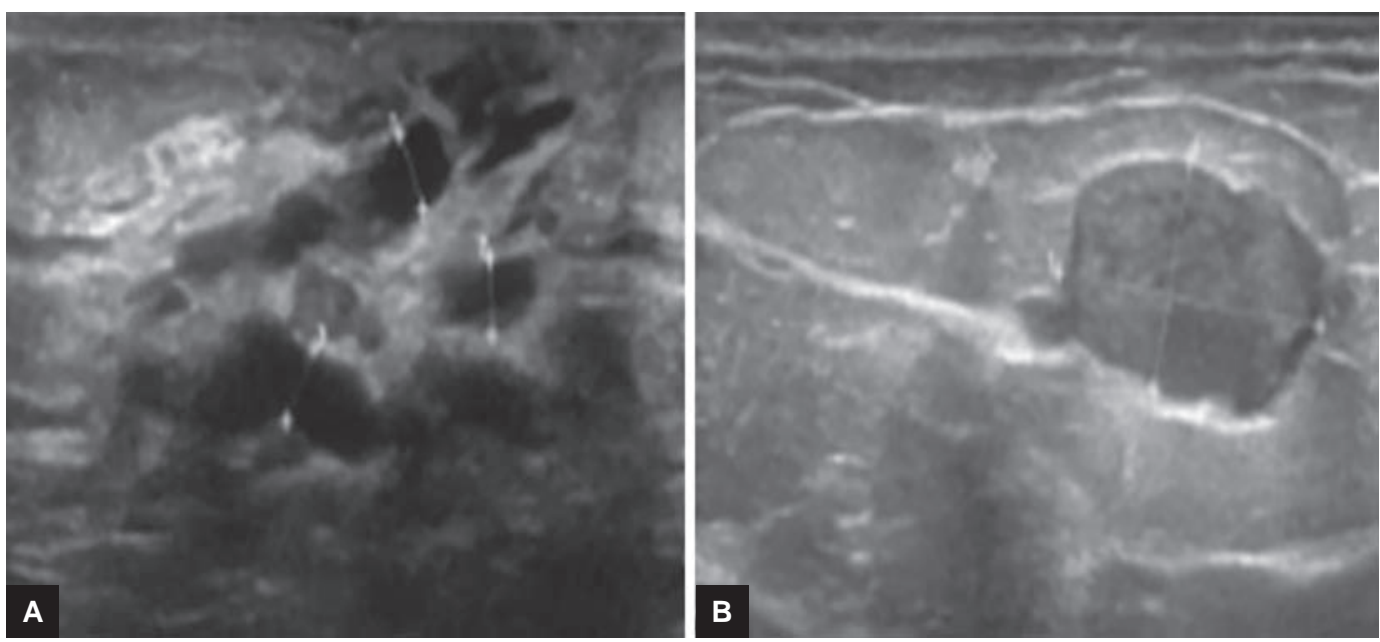

Figs 11A and B: Intraductal papilloma: (A) Sonogram showing dilated retroareolar ducts; and (B) and a well-defined hypoechoic intraductal lesion

The most common cause of bloody nipple discharge is an intraductal papilloma. These are benign ductal neoplasm with a proliferation of epithelial and myoepithelial cells supported by a frond-forming fibrovascular stalk. On mammography it appears as dilated duct, with round or oval circumscribed or indistinctly marginated mass with clustered amorphous or punctate calcifications (Ca++).

On USG, well-circumscribed lobulated intraductal mass in the periareolar region with dilated ducts around the lesion is the most common finding (Fig. 11). They may appear hypervascular at color Doppler assessment. ${ }^{13}$ Ducts may contain echogenic fluid if bleeding has occurred. Current literature indicates excision appropriate for papillomas, even if benign on cytology. According to two recently published studies, there is a 3.1 or $5 \%$ increase in the rate of papillary ductal carcinoma in-situ at surgical excision. ${ }^{14,15}$ Characteristics suggesting an increase in upgrade potential include lesion size $(>1 \mathrm{~cm})$, lesion location $\left(>3 \mathrm{~cm}\right.$ from the nipple), and patient's age ( $>50$ years). ${ }^{15}$

\section{ASYMMETRY}

Global asymmetry means a greater volume of breast tissue in comparison to opposite breast. Focal and global glandular hyperplasia is one of the important cause of palpable nodularity, especially in young females with dense breast (Fig. 12). Radiologist plays a significant role in breast asymmetry of size, density, glandular component whether global or focal and to assure the patient about the benign nature of these pseudolumps. They can further guide for short-term interval follow-up as per treatment response to symptoms. Asymmetries can be taken as benign when not associated with any mass, suspicious calcification and architecture distortion on a mammogram. The USG is also useful in the workup of subtle asymmetries, as it can help diagnose or exclude the presence of an underlying mass.

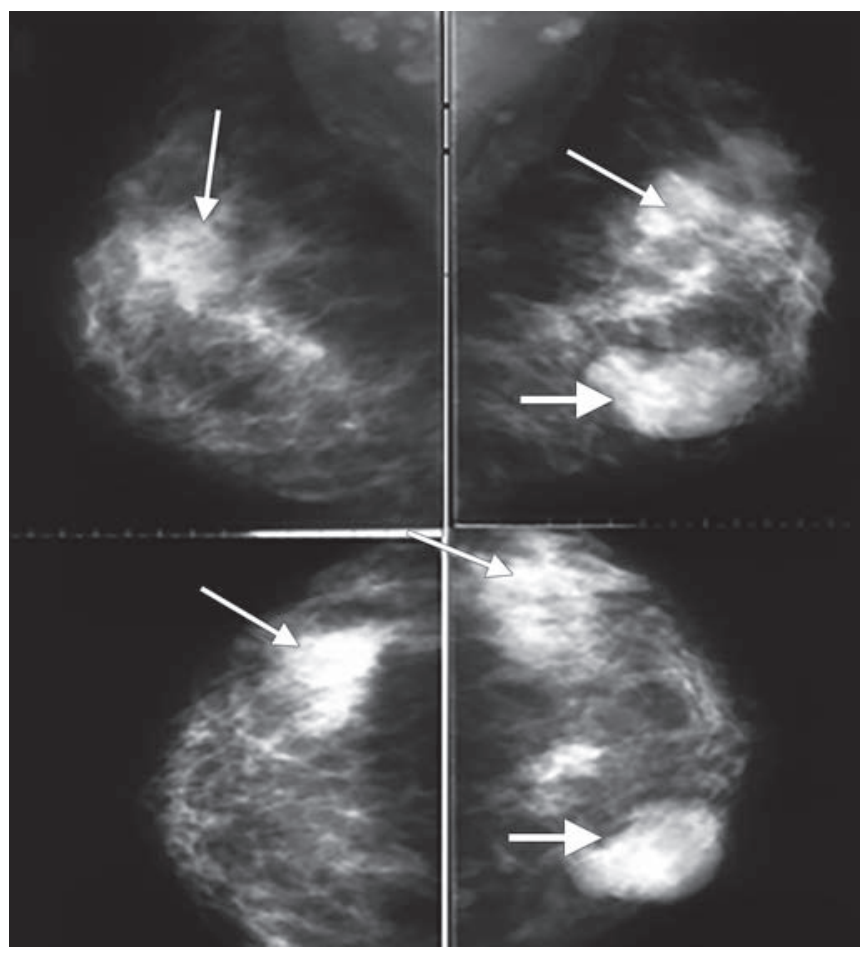

Fig. 12: The MLO and CC view mammogram showing globular asymmetric glandular predominance in bilateral superiolateral breast corresponding to palpable painful nodularity (thin arrow) in otherwise mixed predominantly fibrofatty parenchyma and incidental fibroadenoma in left inferiomedial quadrant (thick arrow)

Approximately 3\% of mammographic examinations reveals global asymmetry. ${ }^{16}$ It is usually due to normal variations or hormonal influence but may be significant when it corresponds to a palpable abnormality. We have found a significant correlation with asymmetric glandular hyperplasia and diffuse fatty breast enlargement in patients with deranged thyroid profile.

Fibrocystic changes in the breast are physiologic alterations, commonly seen in the third decade of life, although such changes may also present in late adolescence. ${ }^{17}$ 
Patients present with cyclically tender breasts feel nodular at palpation. ${ }^{11}$ It has no specific USG findings, ranging from variable-size multiple cysts, ductal dilatation, or fibrous tissue presenting as echogenic foci with posterior acoustic enhancement. Ultrasound needs to be done in follow-up to see any change in size and related complication if any.

\section{BREAST INFECTION}

Ultrasound has the added advantage of diagnosing infective condition due to tenderness associated with mammographic compression and suboptimal image quality.

Mastitis and abscess present as tender, palpable mass near the nipple. Mammography is hard to perform in these patients due to tenderness. It could be a consequence of mammary duct obstruction or ectasia, cellulitis, an immunocompromised state, or nipple injury. ${ }^{18-20}$ The central or subareolar area is a common location for an abscess. ${ }^{19}$ Ultrasound is helpful in differentiating mastitis from abscess. ${ }^{21}$ Mastitis, on sonography, can have both decreased echogenicity (early phlegmon) and increased echogenicity (usually due to edema of the fatty tissue). An increase in vascularity is usually present on color Doppler imaging. Enlarged reactive lymph nodes are frequently there. An abscess can be diagnosed when a complex round, oval, or irregular hypoechoic collection is evident, with reduced central flow on color Doppler. ${ }^{21,22}$

Depending on the time of presentation an abscess may have a sharp or ill-defined outline. Ultrasound findings show a hypoechoic complex mass (Fig. 13), often with a thick wall and peripheral flow on color Doppler seen. ${ }^{23}$ Sonography may aid in guidance of needle for aspiration of the abscess. These lesions show complex morphology on USG with surrounding edema and are given a higher BI-RADS category to avoid missing an underlying pathology. The USG also assesses communication to the ductal system if any or extension on chest wall beside adding in diagnostic/therapeutic aspiration.

\section{Breast Tuberculosis}

The incidence of tuberculosis, in general, is still quite high in India. Direct extension from adjacent structures, such as infected rib, costochondral cartilage, sternum, shoulder joint, and even through the chest wall from tuberculous pleurisy or via abrasions in the skin can occur. ${ }^{24}$ Mammography is not much useful, and US provides better imaging to differentiate abscess. Computed tomography scan not only shows the lung parenchymal changes but also can be used to define the track for the spread and intrathoracic extension/communication if any. We have found four cases of breast tuberculosis as abscess, mastitis and two with intrathoracic/extrapleural pleural extension with contiguous rib involvement. Discharging sinuses/direct fistulous tract with the pleura or a destroyed rib-fragment in the abscess can also be seen (Fig. 14). On USG and further evaluation by contrast-enhanced computed tomography chest to see any associated pleuroparenchymal involvement.

\section{ROLE OF RADIOLOGIST BEYOND IMAGING}

Breast Imaging Reporting and Data System Lexicon criteria and recommendation for further management depend on imaging features, not palpability. Thus, the radiologist should decide the type of investigation to start with, and further workup after analysis of imaging features and assignment of final BI-RADS categorized. The modality to
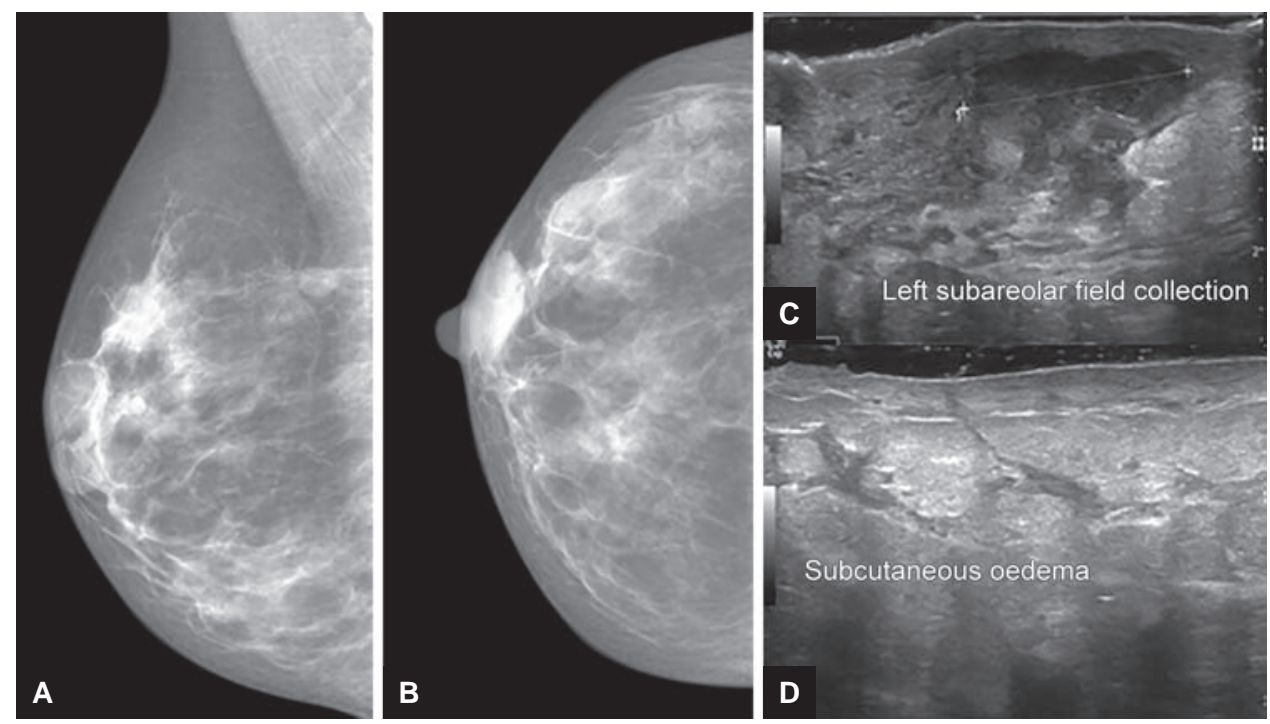

Figs 13A to D: Abscess: (A) Mammogram MLO: (B) CC; (C) view showing retroareolar density with corresponding ultrasonography showing hypoechoic retroareolar collection; and (D) surrounding subcutaneous edema 

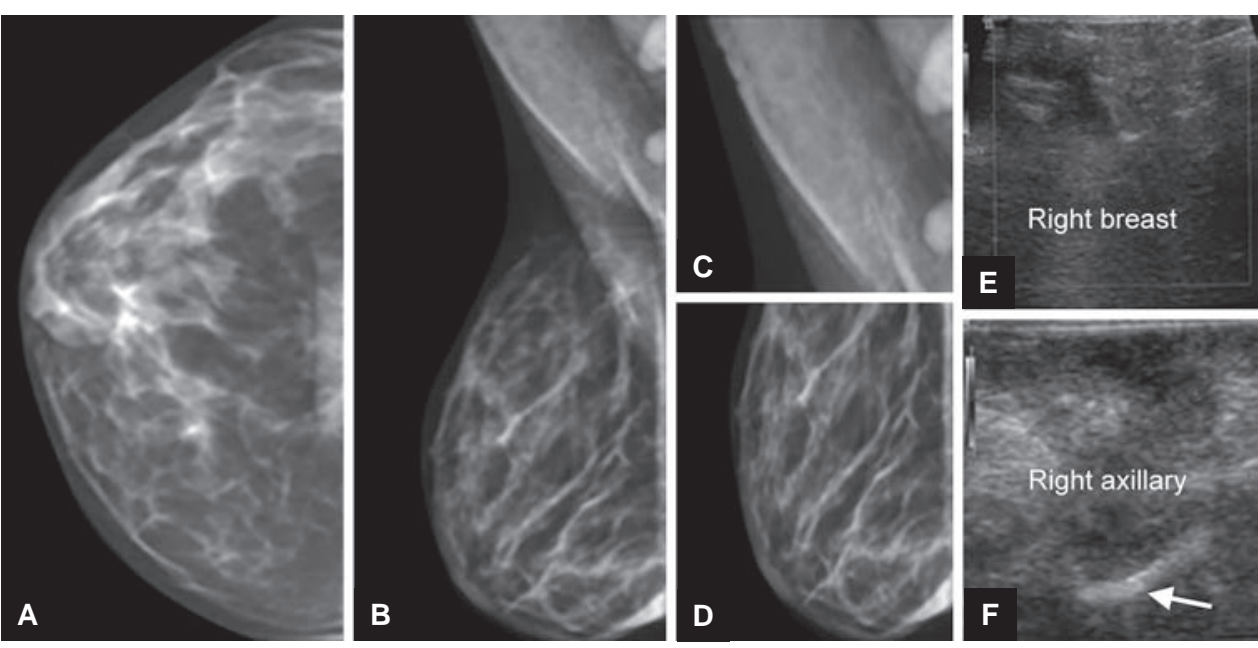

Figs 14A to F: Breast tuberculosis-CC (A) and MLO; (B) mammogram shows assymetric thickening of fibrovascular interstitium with haziness in adjacent fat and thickening of inframammary fold abutting adjacent sinus; (C) shows zoomed view showing lobulated lymphnodes (arrow); (D) shows thickened inframammary fold(arrow). Sonogram (E,F) shows heterogenous echogenic thickening of fat with fluid tracking along the eroded rib margin.

evaluate a breast pathology should always be considered taking many factors into account like radiation, tenderness in infection, lesion in children, and young breast. Ultrasound should be the initial modality to evaluate breast in young females not only due to fibroglandular breast but also to avoid unnecessary mammography and biopsy in the benign lesion (cystic lesion) because biopsy will do more harm to growing tissue than benefit. Mammography should be the first imaging in fatty breast and also for the screening of cancers in various age groups as it is more sensitive to pick up microcalcification and architecture distortion.

The current cancerophobic climates surrounding breast disease affect the proper sequence of imaging modalities and follow-up keeping the invasive methods over other. It is apparent that many patients, with the unanimity of their surgeons and primary physicians, are insisting on undergoing biopsy of nonpalpable lesion and pseudonodules despite the contrary recommendations of the radiologist. Recommendations to perform biopsy over 6-month follow-up often relate to factors other than the estimated probability of the imaged lesion being cancer. It does not reflect inexperience on the part of an investigation but a part of anxiety which surrounds the patient and the physician to rule out malignancy. Most of such lesions which can be followed up undergo biopsy although many of them turn out benign but later on needs follow-up. But a significant disadvantage lies in biopsy of such lesions is the increase in false negative rates resulting in loss of follow-up of patients owing to ignorance of the cause of disease progression.

To somewhat rectify this situation, a competent and confident radiologist or radiology-based breast health educator should discuss the advantages and disadvantages of mammographic surveillance with the patient while she is still in the radiology department.

Radiologists are more involved in clinical assessment during sonomammographic evaluation of the patient and can counsel them.

\section{CONCLUSION}

The study concludes the spectrum of benign lesions with variants and some rare pathologies. The role of a radiologist is paramount in diagnosing, counseling, and referring the breast lesion for proper management by a primary physician.

\section{REFERENCES}

1. Ellis RL. Sonographic confirmation of a mammographically detected breast lesion. AJR Am J Roentgenol 2011 Jan;196(1):225-226.

2. Berg WA, Woel BS. Mammographic-sonographic correlation. Ultrasound Clin 2007; 1(4):567-591.

3. Brem RF, Lenihan MJ, Lieberman J, Torrente J. Screening breast ultrasound: past, present, and future. AJR Am J Roentgenol 2015 Feb;204(2):234-240.

4. Rinaldi P, Ierardi C, Costantini M, Magno S, Giuliani M, Belli P, Bonomo L. Cystic breast lesions: sonographic findings and clinical management. J Ultrasound Med 2010 Nov;29(11):1617-1626.

5. Stavros AT, Thickman D, Rapp CL, Dennis MA, Parker SH, Sisney GA. Solid breast nodules: use of sonography to distinguish between benign and malignant lesions. Radiology 1995 Jul;196(1):123-134.

6. Stavros, AT. Sonographic evaluation of breast cysts. In: Stavros, AT., editor. Breast ultrasound. Philadelphia: Lippincott Williams \& Wilkins; 2004. p. 276-350.

7. Sanchez R, Ladino-Torres MF, Bernat JA, Joe A, DiPietro MA. Breast fibroadenomas in the pediatric population: common and uncommon sonographic findings. Pediatr Radiol 2010 Oct;40(10):1681-1689. 
8. Tse GM, Niu Y, Shi HJ. Phyllodes tumor of the breast: an update. Breast Cancer 2010;17(1):29-34.

9. Cotran, RS.; Kumar, V.; Robbins, SL. Robbins pathologic basis of disease. 5th ed. Philadelphia: Saunders; 1994. p. 1093-1094.

10. Kushwaha AC, O'Toole M, Sneige N, Stelling CB, Dryden MJ. Mammographic-pathologic correlation of apocrine metaplasia diagnosed using vacuum-assisted stereotactic core-needle biopsy: our 4-year experience. AJR Am J Roentgenol 2003 Mar;180(3):795-798.

11. Cardenosa, G. Breast imaging companion. 2nd ed. Philadelphia: Lippincott Williams \& Wilkins; 2008. p. 38.

12. Pui MH, Movson IJ. Fatty tissue breast lesions. Clin Imaging 2003 May-Jun;27(3):150-155.

13. Brookes MJ, Bourke AG. Radiological appearances of papillary breast lesions. Clin Radiol 2008 Nov;63(11):1265-1273.

14. Chang JM, Moon WK, Cho N, Han W, Noh DY, Park IA, Jung EJ. Management of ultrasonographically detected benign papillomas of the breast at core needle biopsy. AJR Am J Roentgenol 2011 Mar;196(3):723-729.

15. Youk JH, Kim EK, Kwak JY, Son EJ, Park BW, Kim SI. Benign papilloma without atypia diagnosed at US-guided 14-gauge core-needle biopsy: clinical and US features predictive of upgrade to malignancy. Radiology 2011 Jan;258(1):81-88.

16. Kim SM, Park JM. Normal and abnormal US findings at the mastectomy site. Radiographics 2004 Mar-Apr;24(2): 357-365.
17. Chung EM, Cube R, Hall GJ, González C, Stocker JT, Glassman LM. From the archives of the AFIP: breast masses in children and adolescents: radiologic-pathologic correlation. Radiographics 2009 May-Jun;29(3):907-931.

18. West KW, Rescorla FJ, Scherer LR 3rd, Grosfeld JL. Diagnosis and treatment of symptomatic breast masses in the pediatric population. J Pediatr Surg 1995 Feb;30(2):182-186; discussion 186-187.

19. Weinstein SP, Conant EF, Orel SG, Zuckerman JA, Bellah R. Spectrum of US findings in pediatric and adolescent patients with palpable breast masses. Radiographics 2000 Nov-Dec;20(6):1613-1621.

20. Greydanus DE, Matytsina L, Gains M. Breast disorders in children and adolescents. Prim Care 2006 Jun;33(2):455-502.

21. Borders H, Mychaliska G, Gebarski KS. Sonographic features of neonatal mastitis and breast abscess. Pediatr Radiol 2009 Sep;39(9):955-958.

22. Kaneda HJ, Mack J, Kasales CJ, Schetter S. Pediatric and adolescent breast masses: a review of pathophysiology, imaging, diagnosis, and treatment. AJR Am J Roentgenol 2013 Feb;200(2):W204-W212.

23. Gokhale S. Ultrasound characterization of breast masses. Indian J Radiol Imaging 2009 Aug;19(3):422-427.

24. Tewari M, Shukla HS. Breast tuberculosis: diagnosis, clinical features \& management. Indian J Med Res 2005 Aug;122(2):103-110. 